Orehek, E. \& Ferrer, R. (in press). Parent instrumentality for adolescent eating and activity. Annals of Behavioral Medicine.

\author{
Parent Instrumentality for Adolescent Eating and Activity \\ Edward Orehek, Ph.D. ${ }^{1} \&$ Rebecca Ferrer, Ph.D. ${ }^{2}$
}

1. University of Pittsburgh

210 S. Bouquet St.

Pittsburgh, PA 15260

orehek@pitt.edu

phone: 412-624-8267

fax: 412-624-4428

2. National Cancer Institute

rebecca.ferrer@nih.gov

301-852-1167

\title{
Acknowledgements
}

The FLASHE study was funded by the National Cancer Institute under contract number

HHSN261201200039I issued to Westat. The content of this publication does not necessarily reflect the views or policies of the Department of Health and Human Services, nor does mention of trade names, commercial products, or organizations imply endorsement by the US Government. 


\begin{abstract}
Background: Parent-adolescent interactions have health implications for adolescents. Parents can be instrumental to healthy eating by purchasing fruits and vegetables or refraining from purchasing hedonic (low nutrient, high energy-dense) foods. Parents can be instrumental to healthy activity by modeling exercise behavior or discouraging sedentary activities. Purpose: This research leverages theory on goal pursuit within relationships to investigate whether parents are instrumental to adolescent's eating and activity. Methods: Using a national sample of 1556 parent-adolescent dyads, we conducted dyadic analyses to examine whether parent instrumentality (both parent-perceived and adolescent-perceived) for healthy behaviors was associated with adolescent engagement in those behaviors. We examined whether the link between parent instrumentality and adolescent BMI was mediated by parent instrumentality. We also explored whether parent instrumentality was associated with parent behaviors and parent BMI. Results: Greater adolescent-perceived parent instrumentality was associated with greater fruit and vegetable consumption and physical activity, and lower sedentariness. Parent-perceived parent instrumentality was associated with greater adolescent fruit and vegetable consumption, less hedonic eating, and more activity. Mediation modeling suggests that adolescent BMI is partially attributable to parent instrumentality for activity. Instrumental parents also engage in healthier behaviors, some of which in turn are associated with lower parent BMI. Conclusions: Findings have implications for the promotion of healthy eating and activity patterns among adolescents. Parental instrumentality for behavior may be an important target for interventions to improve adolescent health, and interventions may be most successful in facilitating adolescent behavior change if they target both parent- and adolescent-perceived parent instrumentality.
\end{abstract}

Keywords: diet; eating; activity; exercise; parent; adolescent; BMI 


\section{Parent Instrumentality for Adolescent Eating and Activity}

Youth obesity in the United States has tripled since 1970 (1), resulting in a population in which about $20 \%$ of youth are obese, and many more are overweight (2). Contributors to adolescent obesity include a diet low in fruits and vegetables and high in hedonic (i.e., low nutrient, high energy-dense) foods, lack of exercise, and sedentariness (3). Consequently, several Healthy People 2020 objectives target adolescents' eating and point to parental support as critical in predicting or changing behavioral patterns established during youth that have a lasting influence on adult health and disease (4). The present research leverages recent theoretical advances in understanding how relationship partners are instrumental to one another's goals $(5,6)$ to investigate how parent instrumentality may influence adolescent eating and physical activity, and a subsequent marker of physical health that these behaviors contribute to: adolescent body mass index (BMI).

Understanding how parents can be instrumental to their adolescents' eating is important. Adolescents may rely on their parents to access healthy food (7): some parents may shop for their children, prepare their food, and eat with them. However, some parents may not engage in these activities. When a parent shops for food, prepares food, encourages the consumption of specific foods, or eats these foods together with his or her adolescent, s/he is instrumental to food consumption by becoming part of the way food are consumed. For example, parents can be instrumental to healthy eating by purchasing fruits and vegetables, or by choosing not to purchase caloric but low-nutrient (i.e., hedonic) foods. The present research investigates whether adolescents are more likely to consume foods for which their parent is instrumental. We predict that an adolescent should be more likely to consume foods when a parent is instrumental to their consumption.

Adolescent physical activity behaviors may, in many instances, rely on parent involvement. For example, social norms and expectations play a vital role in adoption of sedentary behaviors (e.g., 8). 
Adolescents in particular may need parent facilitation for certain activities (e.g., transportation to a sports league; 9), and may benefit from their parent's blocking of sedentary activities (e.g., limiting television and videogame time). A parent may be instrumental to an adolescent's activity by encouraging exercise, engaging in exercise activities together with the adolescent, or limiting screen time or availability of electronics that encourage sedentariness. The present research investigates whether adolescents with instrumental parents are more likely to engage in activities facilitated by their parent. As with eating, we predict that an adolescent should be more likely to engage in activities for which a parent is instrumental.

Though outside of the parent-adolescent interaction and not specific to instrumentality, research on relationships is consistent with these predictions. People who are satisfied in their romantic relationships (10) and who have a supportive partner (11) generally perform better in their goal pursuit, including health behaviors. Moreover, research on health behavior change suggests that having people who are generally supportive of healthy behaviors, including eating and physical activity, are more likely to engage in those behaviors (12-14). Social support for eating and activity may be particularly important for youth (15).

Although generally in line with the present predictions, each of these studies stopped short of addressing how individuals can help close others achieve their goals, and in particular how parents may be instrumental to consequential adolescent health behaviors. Research on relationship satisfaction and social support examined general relationship evaluations, or support for goals in general, rather than instrumentality to specific goals. Adolescents are also more likely to eat healthy foods and avoid unhealthy foods to the extent that their parents model these behaviors (e.g., 16, 17). This research on parent modeling focuses on how parents serve as examples for healthy behaviors, but has not considered other ways parents could be instrumental (e.g., encouraging eating or activity behavior, purchasing 
health foods, providing transportation to sports activities, limiting screen time, etc.). Thus, the present research is poised to fill an important gap in knowledge concerning the way in which parent instrumentality for specific health behaviors may contribute to adolescent enactment of those health behaviors.

We leveraged a large national study of parent-adolescent dyads (18) to examine parent instrumentality to adolescent eating and activity behaviors. The study included assessments of parent instrumentality for fruit and vegetable consumption, hedonic food consumption, physical activity, and sedentariness using both adolescent and parent reports. We conducted actor-partner interdependence modeling analyses to examine unique variance in adolescents' and parents' perceptions of parent eating and activity goals in predicting adolescents' (and parents') eating and activity behaviors, as well as adolescents' (and parents') BMI. Our primary predictions concerned adolescents' eating and activity behaviors, given that the instrumentality predictors are asymmetrical (i.e., both parents and adolescents reported parent instrumentality, as opposed to each reporting the other's instrumentality).

The study design allows us to investigate whether parent actions that are visible to adolescents (i.e., reported by them) as well as actions that are invisible to adolescents (i.e., reported by parents but not reported by adolescents) each predict adolescent behaviors (e.g., 19, 20), For example, when a parent purchases fruits and vegetables, the adolescent may be aware that the parent has done so (visible) or may not explicitly acknowledge that the parent has facilitated their consumption (invisible). We predicted that both visible (i.e., adolescent-perceived) and invisible (i.e., parent-perceived, controlling for adolescent-perceived) instrumentality would account for unique variance in adolescent eating, activity, and BMI.

In addition, we conducted exploratory analyses testing the possibility that parent instrumentality for adolescents' health goals may be associated with parents' own behavior. For example, if parents 
purchase healthy foods for adolescents, or make a point to model healthy activity, these actions (although perhaps not intended as self-improvement) may also improve parent behaviors (and BMI). However, we acknowledged that it was also possible that parent instrumentality would not translate to their own behavior because the focus is on their children rather than on themselves, or that parent instrumentality would have a negative association with parent behavior because encouraging or helping one's adolescent may serve as a way to excuse one's own poor health behaviors. We are not aware of any previous data examining these possibilities, and thus did not have a directional hypothesis.

Our specific hypotheses were as follows:

Hypothesis 1a: Adolescent-perceived parent instrumentality will be associated with more adolescent fruit and vegetable $(\mathrm{F} \& \mathrm{~V})$ consumption and exercise and less adolescent hedonic food consumption and sedentariness. This hypothesis will be assessed by examining direct actor paths among adolescent-perceived parent instrumentality and these adolescent outcomes.

Hypothesis 1b: Associations of adolescent-perceived parent instrumentality and adolescent BMI will be indirectly associated via adolescent eating and activity behaviors. This hypothesis will be assessed by examining indirect actor paths among adolescent-perceived parent instrumentality and adolescent BMI via adolescent behaviors.

Hypothesis 2a: Parent-perceived self-instrumentality will be associated with more adolescent F\&V consumption and exercise and less adolescent hedonic food consumption and sedentariness. This hypothesis will be assessed by examining direct partner paths between parent-perceived selfinstrumentality and these adolescent outcomes.

Hypothesis 2b: Associations of parent-perceived self-instrumentality and adolescent BMI will be indirectly associated via adolescent eating and activity behaviors. This hypothesis will be assessed by 
examining indirect partner paths among parent-perceived self-instrumentality and adolescent BMI via adolescent behaviors.

Exploratory Hypothesis 3a: Parent-perceived self-instrumentality may be associated with parent F\&V consumption and exercise and parent hedonic food consumption and sedentariness. This hypothesis will be assessed by examining direct actor paths among parent-perceived self-instrumentality and these parent outcomes.

Exploratory Hypothesis 3b: Associations of parent-perceived self-instrumentality and parent BMI may be indirectly associated via parent eating and activity behaviors. This hypothesis will be assessed by examining indirect actor paths among parent-perceived self-instrumentality and parent BMI via parent behaviors.

Exploratory Hypothesis 4a: Adolescent-perceived parent instrumentality may be associated with parent F\&V consumption and exercise and parent hedonic food consumption and sedentariness. This hypothesis will be assessed by examining direct partner paths between adolescent-perceived parent instrumentality and these parent outcomes.

Exploratory Hypothesis 4b: Associations of adolescent-perceived parent instrumentality and parent BMI will be indirectly associated via parent eating and activity behaviors. This hypothesis will be assessed by examining indirect partner paths among adolescent-perceived parent instrumentality and parent BMI via parent behaviors.

\section{Method}

\section{Participants and Procedure}

This study uses data collected in the National Cancer Institute (NCI)'s Family Life, Activity, Sun, Health, and Eating (FLASHE) study (http://cancercontrol.cancer.gov/brp/hbrb/flashe.html). FLASHE is a web-based, cross-sectional study of parent-adolescent dyads, and was administered 
through Westat, Inc. Data were collected between April and October 2014. Parent participants were recruited to the study through the Ipsos Consumer Opinion Panel, using sample balancing methods (18). Sample distributions closely match the U.S. population on the following demographics: gender of the adult panel member, census division, household income, household size, and race/ ethnicity. Panelists were eligible if they were 18 years of age or older and lived with at least one child between the ages of 12 and 17 at least half-time.

The final set of participants used in these analyses were 1556 parent-adolescent dyads $(N=$ 3112) who completed all measures used in analyses. The sample was $63 \%$ female (75\% of parents, $50 \%$ of adolescents). Parent mean age was $43.66(S D=7.91)$ and adolescent mean age was $14.45(S D=$ 1.62). Full sociodemographic characteristics for parents, adolescents, and the full sample are in Table 1.

The study was conducted online, and surveys were emailed to participants. Additional details about the study methodology and item development are available elsewhere $(18,21)$. The study was approved by the NCI Special Studies Institutional Review Board (SSIRB), and Westat, Inc.'s Institutional Review Board (IRB).

\section{Measures}

All measures were similarly assessed in both parents and adolescents to facilitate dyadic analyses. Measures were developed based on extensive reviews of the literature, expert consensus, and cognitive testing. Cognitive testing is a process for refining survey items prior to administration $(22,23)$. During the cognitive testing process, individuals (selected based on expected similarity to participants for the survey being developed, with attention to socioeconomic, educational, and racial diversity) were brought into a lab and asked to go through the survey out loud. They were asked to describe all of their thoughts while answering the survey, including thoughts about the item and its interpretation, what they are thinking when reading the item, how they selected their answer, and what their answer means. This 
process helped to ensure that items were being interpreted in the intended way and that they were written at an appropriate reading level. It also helps to refine the way that questions are asked, as well as the response items provided. The cognitive testing process for FLASHE is described in more detail by Nebeling and colleagues (21). Participants completed the surveys online, while at home. Participants were instructed to complete the questionnaires alone and without help. All measures in the FLASHE study, including sources and origin of development information, are available elsewhere (21).

Parent instrumentality. Parent instrumentality variables were drawn from published studies (2429; see 21) and all had response options: 1 (strongly disagree) through 5 (strongly agree). Adolescentperceived parent instrumentality $(\alpha=.81)$ and parent-perceived self-instrumentality $(\alpha=.79)$ for fruit and vegetable consumption (F\&V consumption) were both assessed with 7-item scales. Example items include: "My parents buy fruits and vegetables for me/ I buy fruits and vegetables for my teenager;" "My parents try to eat fruits and vegetables when I am around/ I try to eat fruits and vegetables when my child is around" and "My parents encourage me to try different kinds of fruits and vegetables/ I encourage my teenager to try different kinds of fruits and vegetables.” Higher scores indicate greater instrumentality for fruit and vegetable consumption.

Adolescent-perceived parent instrumentality $(\alpha=.74)$ and parent-perceived self-instrumentality ( $\alpha=.70$ ) for avoiding hedonic food consumption were both assessed with 7 -item scales. Example items included "If I have a bad day, my parents let me have junk food or sugary drinks to make me feel better/ If my teenager has a bad day, I let him or her have junk food or sugary drinks to feel better" (reversecoded); “My parents don't buy a lot of junk food or sugary drinks for me/ I don’t buy a lot of junk food or sugary drinks for my teenager;" and "My parents try to avoid eating junk food or drinking sugary drinks when I am around/ I try to avoid eating junk food or drinking sugary drinks when my teenager is around. Higher scores indicate greater instrumentality for avoiding hedonic food consumption. 
Adolescent-perceived parent instrumentality $(\alpha=.81)$ and parent-perceived self-instrumentality $(\alpha=.81)$ for exercise were both assessed with 6-item scales. Example items include: "My parents take me places where I can be physically active/ I take my teenager places where he or she can be physically active;" "My parents make me exercise or go out and play/ I make my teenager exercise or go out and play;" and "My parents try to be physically active when I'm around/ I try to be physically active when my teenager is around." Higher scores indicate greater instrumentality for exercise.

Adolescent-perceived parent instrumentality $(\alpha=.56)$ and parent-perceived self-instrumentality ( $\alpha=.73)$ for preventing sedentariness were both assessed with 7-item scales. Examples include: "If I have a bad day, my parents let me have screen time to make me feel better/ If my teenager has a bad day, I let him or her have screen time to feel better" (reverse-coded); "My parents take me places where I can play video games, watch movies, etc./ I take my teenager places where he or she can play video games, watch movies, etc." (reverse-coded) and "My parents try to limit their screen time when I am around/ I try to limit my screen time when my teenager is around." Higher scores indicate greater instrumentality for preventing sedentariness.

Dietary outcomes. For both adolescents and adults, frequency of foods and beverages were assessed with a 27-item dietary screener, which was adapted from several sources (30-33). The 27-item version of the screener used here has been validated in comparison to in-depth food frequency questionnaires (34), objectively assessed dietary assessments (35), and intensive 24-hour recalls (36). More information about the dietary screener is available elsewhere (33).

The screener assessed frequency of foods eaten and beverages consumed over the past 7 days. For example, participants were asked: "DURING THE PAST 7 DAYS, how many times did you eat FRUIT like apples, bananas, melons, etc.?" Options were: 1: I did not eat [food] during the past 7 days; 2: 1-3 times in the past 7 days; 3: 4-6 times in the past 7 days; 4: 1 time per day; 5: 2 times per day; and 
6: 3 or more times per day. Responses were converted to daily frequencies (never $=0 ; 1$ to 3 times during the past 7 days $=0.29 ; 4$ to 6 times during the past 7 days $=0.71 ; 1$ time per day $=1$; etc.). Fruit and vegetable frequency was comprised of the sum of 3 items reporting consumption of green salad, non-fried vegetables, and fruit. Hedonic food frequency was comprised of the sum of 8 items that reported consumption of sugary cereal, candy and chocolate, fried potatoes, chips, processed meat, cookies and cake, frozen desserts, and fast food.

Adolescent physical activity and sedentariness were assessed with the Youth Activity Profile (YAP; 35). The YAP is a 15-item self-administered, 7-day recall instrument that is used to assess physical activity and sedentariness among adolescents in fourth through $12^{\text {th }}$ grade. Items capture activity during school (e.g., recess, gym, transportation to and from school), as well as activity right after school (e.g., sports practices), and activity in the evening and on weekends. Sedentary items assess time spent watching television, playing videogames, and using the computer, tablets, phones, or other screens. We applied a previously validated algorithm for improving accuracy of the YAP (37). The validation included a sub-sample of adolescents $(n=119)$, who wore accelerometers on their wrists for two weeks prior to completing the YAP. The minutes of activity/ inactivity estimates generated from accelerometer data (based on counts) were regressed on YAP subsection scores, age, and the interaction between the two to generate an algorithm to render the YAP more accurate (i.e., converts YAP scores to accelerometer activity estimates). The algorithm was then applied to independent samples ( $\mathrm{n}=39-51)$ to demonstrate validity (37). Once validated, this algorithm was then applied to all adolescent physical activity and sedentariness behavior YAP scores generated by the FLASHE adolescent sample to generate two refined scores (which were those used in the present analyses), which reflected minutes of activity and sedentariness per week. These procedures are described in detail elsewhere (37). 
Parent physical activity and sedentariness were assessed with a series of four items from the International Physical Activity Questionnaire - Short Form (38-40). Parents were given the following instructions: "We are interested in finding out about the kinds of physical activities that people do as part of their everyday lives. The questions will ask you about the time you spent being physically active in the past 7 days. Please answer each question, even if you do not consider yourself to be an active person. Please think about the activities you do at work, as part of your house and yard work, to get from place to place, and in your spare time for recreation, exercise, or sport." Then, they were asked a series of questions about specific activities: "Think about all the VIGOROUS activities that you did in the PAST 7 DAYS. VIGOROUS physical activities refer to activities that take hard physical effort and make you breathe much harder than normal. Think ONLY about those physical activities that you did for at least 10 minutes at a time. During the LAST 7 DAYS, on how many days did you do VIGOROUS physical activities like heavy lifting, digging, aerobics, or fast bicycling?"; "How much time did you usually spend doing VIGOROUS physical activities on one of those days?"; "Think about all the MODERATE activities that you did in the LAST 7 DAYS. MODERATE activities refer to activities that take moderate physical effort and make you breathe somewhat harder than normal. Think ONLY about those physical activities that you did for at least 10 minutes at a time. During the LAST 7 DAYS, on how many days did you do MODERATE physical activities like carrying light loads, bicycling at a regular pace, or doubles tennis? Do not include walking."; "How much time did you usually spend doing MODERATE physical activities on one of those days?"; "Think about the time you spent WALKING in the LAST 7 DAYS. This includes at work and at home, walking to travel from place to place, and any other walking that you have done solely for recreation, sport, exercise, or leisure. During the LAST 7 DAYS, on how many days did you WALK for at least 10 minutes at a time?"; "How much time did you usually spend WALKING on one of those days?"; The last question is about the time you spent 
SITTING on weekdays during the LAST 7 DAYS. Include time spent at work, at home, while doing course work and during leisure time. This may include time spent sitting at a desk, visiting friends, reading, or sitting or lying down to watch television. During the LAST 7 DAYS, how much time did you spend SITTING on a WEEK DAY?” All items were open-ended, but included appropriate labels for the responses (e.g., days, hours, minutes). Outliers were removed according to the IPAQ protocol (41). Minutes of physical activity per week was computed including vigorous activity, moderate activity, and walking. Minutes of sedentariness per week was also computed.

Body mass index was calculated using parents' and adolescents' self-reported height (in feet and inches) and weight (in pounds), which were converted to centimeters and kilograms for calculations. The survey platform was pre-programmed with "soft" system checks such that when a participant entered a value outside of the biologically plausible range for human height or weight, a pop-up box appeared requiring participants to confirm their response before they could move to the next question. BMI data were also screened post-submission, and outliers beyond what would be biologically plausible for humans were removed (see 42).

Sociodemographic control variables. Analyses controlled for self-reported sociodemographic variables. Race, parent marital status, and parent college education were coded as dichotomous (i.e., dummy-coded), based on variability on these variables in the sample and consistent with the approach in other studies using FLASHE data in dyadic analyses (43). Race was coded white (1)/ non-white (0), and black (1) / non-black (0). Parental marital status was coded married (1)/ non-married (0). Parent education was coded college educated (1)/ less than college educated (0). Gender was coded female (1)/ male (0).

\section{Data Analytic Strategy}


The final analyses presented are the result of a single actor-partner interdependence model (APIM; 44, 45), conducted via structural equation modeling in Mplus version 8. This model simultaneously assessed direct and indirect paths specified in hypotheses. Before running the final model, we conducted preliminary APIM analyses, regressing adolescent and parent BMI on adolescent and parent eating behaviors, respectively (paths from adolescent behavior to parent BMI and parent behavior to adolescent BMI were not evaluated as plausible mediators). Behaviors significantly associated with BMI in this step were evaluated as potential mediators, and indirect paths via behaviors unassociated with BMI were not examined in the final model (46).

In the final model, we examined the direct associations of each of the eight behavioral outcome variables (adolescent eating and activity behaviors parent eating and activity behaviors) with corresponding adolescent- and parent-perceived parent instrumentality behaviors (e.g., both adolescent and parent $\mathrm{F} \& \mathrm{~V}$ consumption were regressed on both adolescent- and parent-perceived instrumentality for adolescent $\mathrm{F} \& \mathrm{~V}$ consumption). We examined the direct associations of both adolescent and parent BMI and all eight instrumentality variables (adolescent-perceived parent instrumentality for adolescent eating and activity behaviors, parent-perceived parent instrumentality for adolescent eating and activity behaviors). Finally, in the same model, we examined the indirect associations of adolescent- and parentperceived instrumentality variables and adolescent and parent BMI, mediated by behavioral variables associated with adolescent or parent BMI identified in the preliminary analyses. Listwise deletion was turned off, and as such, all cases were included unless they had missing data on any exogenous variable. This model includes all four behaviors of interest in a single model. This model uses a full Information Maximum Likelihood (FIML) estimation approach to handling missing values on non-exogenous variables (47). 
This final model initially controlled for parent/ adolescent age, parent/ adolescent gender, parent/ adolescent race (dummy-coded white/ non-white, and black/ non-black), parent/ adolescent ethnicity, parent education (college vs. other), and parent marital status (married vs. other) (described above, and consistent with previous FLASHE analyses; 21, 43). Because parents and adolescents mostly have the same race and ethnicity, the final model included only parent race (dummy-coded white/ non-white, and black/ non-black) and ethnicity.

Modification indices were used to identify parameters that would improve model fit. Modification indices above 50 were examined, and were included in the model if there was theoretical justification for doing so (48). Modification indices suggested the model fit would improve by specifying the following correlations: parent F\&V consumption with parent hedonic food consumption; adolescent F\&V consumption with adolescent hedonic food consumption; parent F\&V consumption with parent exercise; adolescent $\mathrm{F} \& \mathrm{~V}$ consumption with adolescent exercise; parent hedonic food consumption with parent sedentariness; adolescent hedonic food consumption with adolescent sedentariness. Because research suggests that some health behaviors are interrelated (49), we evaluated these suggestions as conceptually appropriate, and incorporated them into the model.

The final model included $n=1353$ dyads who reported data necessary to calculate all instrumentality variables and all included covariates. Of the $n=373$ dyads excluded, $n=359$ were excluded because they lacked data necessary to calculate the instrumentality variables, and only 14 additional dyads were excluded for lacking demographic information. Logistic regression indicated that missing data on instrumentality variables (and thus exclusion from modeling) was not associated with parent/ adolescent age, parent/ adolescent gender, parent/ adolescent race (dummy-coded white/ nonwhite, and black/ non-black), parent/ adolescent ethnicity, parent education (college vs. other), or parent 
marital status (married vs. other) (all $p \mathrm{~s}>$.222). STDYX beta estimates (an effect size estimate of a oneunit standard deviation of each predictor) and their $95 \%$ confidence intervals are reported.

\section{Results}

\section{Descriptive Statistics}

Descriptive statistics are reported in Table 2. T-tests comparing average responses revealed that parents perceived themselves as more instrumental to each of the four behaviors than adolescents reported $(p s<.001)$. This may indicate that parents are instrumental in ways that adolescents do not detect, and/or it may indicate that parents are optimistically biased in reporting their own instrumentality. Both parents and adolescents consume more hedonic foods than they consume fruits and vegetables $(p s<.001)$. Parents report engaging in more sedentary behaviors than exercise, whereas adolescents report engaging in more exercise than sedentary behaviors $(p s<.001)$.

\section{Main Effects Models}

Preliminary Analyses. When considered as a suite of behaviors, included together in APIM (and considering only actor associations for the purpose of identifying plausible mediators), adolescent exercise and sedentariness were associated with adolescent BMI, and parent F\&V consumption, hedonic food consumption, and exercise were associated with parent BMI (See Table 3). These were included as potential mediators in the final model.

Main analyses. Results from the final model are found in Table 4, and are described below, ordered by hypothesis. Model fit was good, $\chi 2(108)=371.70, \mathrm{p}<.001 ; \mathrm{CFI}=0.996 ; \mathrm{RMSEA}=0.042$, $95 \% \mathrm{CI}=0.038,0.047, \mathrm{p}-\mathrm{close}<.001$

Hypothesis 1a: Adolescent-perceived parent instrumentality was associated with greater adolescent $\mathrm{F} \& \mathrm{~V}$ consumption and exercise, as well as lower adolescent sedentariness; however, it was unassociated with adolescent hedonic food consumption. 
Hypothesis 1b: Adolescent-perceived parent instrumentality was indirectly associated with lower adolescent BMI via greater adolescent exercise; however, mediation via adolescent sedentariness was not significant (and adolescent eating behaviors were unassociated with adolescent BMI)

Hypothesis 2a: Parent-perceived self-instrumentality was associated with greater adolescent F\&V consumption and exercise, and less hedonic food consumption. However, it was unassociated with adolescent sedentariness.

Hypothesis $2 b$ : Parent-perceived self-instrumentality was indirectly associated with lower adolescent BMI via greater adolescent exercise; no mediation via adolescent sedentariness emerged (and adolescent eating behaviors were unassociated with adolescent BMI).

Exploratory Hypothesis 3a: Parent-perceived self-instrumentality was associated with greater parent fruit and vegetable consumption, and lower parent hedonic food consumption and sedentariness; however, it was unassociated with parent exercise.

Exploratory Hypothesis 3b: Parent-perceived self-instrumentality was indirectly associated with lower parent BMI via lower parent hedonic food consumption; however, mediation via F\&V and hedonic food consumption was not significant (and parent exercise was unassociated with parent BMI).

Exploratory Hypothesis 4a: Adolescent-perceived parent instrumentality was associated with greater parent $\mathrm{F} \& \mathrm{~V}$ consumption, and more exercise; no other direct partner associations emerged.

Exploratory Hypothesis 4b: Adolescent-perceived parent instrumentality was indirectly associated with parent BMI via parent sedentariness.

Although we did not have any hypotheses regarding the direct paths from parent instrumentality to adolescent and parent BMI, some significant effects emerged. Parent perceived self-instrumentality for adolescent hedonic food consumption and exercise was associated with greater adolescent BMI. Adolescent perceived parent instrumentality for exercise was also associated with greater adolescent 
BMI. Adolescent perceived parent instrumentality for avoiding sedentary behaviors was associated with lower adolescent BMI. Finally, adolescent perceived parent instrumentality for exercise was associated with lower parent BMI.

\section{Discussion}

In accordance with recent theoretical advances on interpersonal processes in goal pursuit $(5,6$, 50), the present analyses demonstrate in a large national sample of U.S. parent-adolescent dyads that adolescents with instrumental parents (perceived by at least the parent or adolescent) eat more fruits and vegetables, eat fewer hedonic foods, engage in more physical activity, and adopt fewer sedentary behaviors. Moreover, parent instrumentality was indirectly associated with lower adolescent BMI via adolescent exercise. In addition, parents who are instrumental to their adolescent (perceived by at least the parent or adolescent) consume more fruits and vegetables, eat fewer hedonic foods, and engage in more exercise, and adopt fewer sedentary behaviors. In addition, parent instrumentality was indirectly associated with lower adolescent BMI via exercise behaviors. Parent instrumentality was indirectly associated with lower parent BMI via hedonic food consumption and sedentary behaviors. These findings suggest that instrumental parents facilitate both adolescent and parent health behaviors and outcomes, which supports recommendations in Healthy People 2020 to examine and target parental support as a predictor of adolescent eating and activity behaviors to improve their weight and future health (4). Below we discuss in more detail specific data patterns, limitations of the present research, potential moderators, and implications of this work.

\section{Parent Instrumentality and Health Behaviors}

Adolescent behavior. Adolescent-perceived parent instrumentality was associated with healthy eating and activity (but not hedonic foods). In addition, adolescent-perceived parent instrumentality was indirectly associated with lower BMI via adolescent exercise. In addition, parent-perceived 
instrumentality was associated with adolescent healthy eating and activity (but not sedentariness), and indirectly associated with lower BMI via exercise. These links support the notion that both visible (i.e., adolescent-perceived) and invisible (i.e., parent-perceived, controlling for adolescent-perceived) instrumentality predict adolescent behavior $(19,20)$. Fruit and vegetable consumption and physical activity were associated with both parent and adolescent perceived parent instrumentality, suggesting, (a) that the link between parent instrumentality and these behaviors is robust, and (b) that both visible and invisible sources of instrumentality are associated with these behaviors. Hedonic food consumption was associated with parent instrumentality as perceived by parent but not adolescent. This could be because (a) the link between parent instrumentality and adolescent hedonic eating is not robust, or (b) hedonic food consumption is driven more by invisible influences. When parents opt against purchasing hedonic foods, they may do so intentionally, but such actions may occur outside the awareness of adolescents. This seems plausible because the absence of something (hedonic foods) should be difficult to detect, or easy to go unnoticed. Adolescent perceived parent instrumentality was associated with sedentary behaviors, but parent perceived instrumentality was not. This could be because (a) the link between parent instrumentality and adolescent sedentariness is not robust, or (b) adolescent sedentariness is more strongly associated with visible parent instrumentality. The latter explanation seems plausible in light of the fact that the main way for a parent to control sedentariness is to set explicit restrictions on such behavior, or otherwise discourage it. It seems that this represents a set of behaviors that is likely to be visible (rather than invisible) to the adolescent. Additional research is needed to more fully explore each of the possibilities raised regarding parent instrumentality for adolescent behaviors.

Although we did not have any hypotheses regarding a direct effect of parent instrumentality on adolescent BMI, we found a positive direct path from parent-perceived and adolescent perceived parent 
instrumentality for exercise on adolescent BMI, and a positive direct path from parent perceived instrumentality for hedonic eating on adolescent BMI. Although speculative, and requiring additional research to confirm, we tentatively interpret this to suggest that parents are more likely to be instrumental to adolescent exercise and hedonic eating when they have adolescents who have higher BMI. We also found a negative direct path from adolescent perceived parent instrumentality for sedentary activities predicting lower BMI, suggesting that adolescents who are aware of parent attempts to limit their sedentary behaviors have lower BMI.

Parent behavior. Also notable is that parent instrumentality was found to be beneficial for parent health behaviors. Specifically, adolescent-perceived parent instrumentality was associated with greater parent fruit and vegetable consumption and more parent exercise. In addition, parent-perceived self-instrumentality was associated with parent healthy eating and sedentary behaviors (but not exercise). Parent perceived self-instrumentality was indirectly associated with lower parent BMI via less hedonic food consumption. Adolescent perceived parent instrumentality was indirectly associated with lower parent BMI via less sedentary activity.

Previous research has found that support providers benefit psychologically by caring for others (51), and these findings suggest that they may also experience positive consequences when it comes to their own behavior. The most robust link was between parent instrumentality (perceived by the parent and adolescent) and fruit and vegetable consumption. It may be that being instrumental toward fruits and vegetables is an especially good way for parents to help their own behaviors because they adopt the behaviors themselves. Parent perceived self-instrumentality was also associated with less parent hedonic eating and lower parent sedentariness. We also found that parent perceived instrumentality for hedonic eating was associated with less parent hedonic eating. Although these associations for between parent instrumentality and these behaviors was only found for one person's perception (only the adolescent or 
parent), these results suggest that parent instrumentality may be associated with healthy behaviors overall. Although we did not have a hypothesis regarding direct paths, we found that adolescent perceived parent instrumentality for exercise was associated with lower parent BMI.

BMI. We found an indirect association between parent instrumentality and both adolescent and parent BMI via some of the health behaviors. This suggests that understanding social processes that contribute to health behaviors is an important avenue for research, and that instrumentality of a partner is an important way of thinking about how social relationship contribute to health $(5,6)$. Some behaviors were more strongly associated with BMI than others. Adolescent exercise and sedentariness were associated with adolescent BMI, and parent fruit and vegetable consumption, hedonic food consumption, and sedentariness were associated with parent BMI. Thus, across both parents and adolescents, it seems that sedentariness was associated with BMI. For parents, but not adolescents, fruit and vegetable consumption and hedonic eating was associated with BMI. This difference may indicate that diet is more strongly associated with BMI for adults than adolescents, or may indicate that it is less reliable as a predictor of BMI. Adolescent, but not parent, exercise was associated with BMI. This may indicate that exercise is more strongly associated with BMI for adolescents than adults, or may indicate that it is less reliable as a predictor of BMI. Finally, the link between parent instrumentality (as perceived by both adolescent and parent) and BMI was mediated by adolescent exercise. Parent instrumentality's link to parent BMI was mediated by parent hedonic eating and sedentariness. This pattern of mediation effects suggests that the link between parent instrumentality and BMI of both adolescent and parent is partially attributable to health behaviors. However, inconsistencies in the specific behaviors suggests that additional research is needed before strong inferences can be made regarding the link between health behaviors and BMI.

\section{Implications for Theory and Intervention}


These findings advance social psychological theory on interpersonal processes in goal pursuit and dyadic processes involved in self-regulation $(5,6,50)$. While previous research has examined ways in which having a relationship partner who serves as a means to goals influences one's evaluations of that partner (52-55), research has yet to investigate the influence of partner instrumentality on behavior. This marks an important gap in understanding how relationship partners are instrumental to consequential goals: healthy eating (i.e., greater fruit and vegetable and less hedonic food consumption) and activity (i.e., greater physical activity and less sedentariness). Parent-adolescent relationships are particularly well-suited for investigation of this theoretical perspective because instrumentality in this type of dependent relationship should have especially important consequences. Without an instrumental parent, an adolescent can literally go without access to (healthy) food or transportation to an activity (e.g., 7, 9). Thus, the present research fills this important gap in knowledge by demonstrating that parent instrumentality to adolescents' healthy eating and physical activity is associated with greater engagement in these behaviors and, in turn, that these health behaviors are associated with lower BMI. An important direction for future research is to investigate whether married (or cohabiting) partners influence each other via instrumentality in the same way.

This research suggests that interventions targeting individual behavior change should place greater emphasis on important social relationships. Specifically, it suggests that a support provider's instrumentality to a specific behavior is associated with that behavior, and therefore suggests that it would be worth investigating whether changes in Partner A's instrumentality lead to partner B's behavior change. These results also suggest a potential unintended benefit for the support provider, namely, that the provider who is instrumental may exhibit healthier behavior.

\section{Limitations and Directions for Future Research}


One limitation is reliance on self-report. This limitation is offset by the use of (a) multiple ratings of parent instrumentality (by parents and adolescents), (b) standard dietary screeners that have been validated against food frequency questionnaires, diet records, and 24-hour recalls, (c) a measure of adolescent activity that was validated against accelerometer data, and (d) system checks for BMI. However, future research attempting to replicate these findings could include objective measurements of eating, activity, and BMI.

A second limitation is that the data are correlational, limiting our ability to make causal inferences. Future research should investigate whether experimentally manipulating perceived partner instrumentality influences motivation to engage in behaviors and behavior change. The potential for the present hypotheses to be translated into interventions rests on such causal pathways, and their malleability.

\section{Potential Boundary Conditions and Moderators}

Future research could explore potential moderators of these effects. For example, interpersonal closeness among parent-adolescent dyads was not measured, which might modify results. In addition, analyses did not consider differences in access to and availability of fruits and vegetables (based on neighborhood environment or season), adolescent peer group, or other socio-structural influences. While the present investigation assessed parent instrumentality, it did not investigate potential barriers to instrumentality, such as access to healthy foods, time demands placed on the parent, or other resources the parent may need in order to be instrumental. It also did not investigate whether environmental or peer influences support or oppose parent attempts to be instrumental. For example, future research could take a social ecological perspective (56) to investigate how interpersonal influences (specifically, partner instrumentality) interacts with the local community (e.g., access to fast food), school environment (e.g., 
physical education and school food programs), and peer instrumentality in facilitating eating and activity behaviors.

\section{Conclusion}

These results suggest that adolescents with parents who are instrumental to healthy eating and physical activity are more likely to engage in healthy behaviors. In addition, adolescent activity is associated with lower adolescent BMI. These findings have implications for interventions designed to improve adolescent health trajectories by targeting eating and activity behaviors. Specifically, these data suggest that encouraging parents to engage in behaviors that are instrumental to their adolescents' healthy eating and activity behaviors has the potential to improve adolescent health behaviors, reduce their BMI, and improve their future health trajectories, in line with Healthy People 2020 recommendations (4). Moreover, these findings suggest that interventions targeting parent instrumentality for adolescent health behaviors may be more efficacious to the extent that they focus on both instrumentality that is apparent to the adolescent (i.e., visible instrumentality), as well as instrumentality that may not be apparent to the adolescent but is apparent to the parent (i.e., invisible instrumentality). 
Table 1. Sociodemographic and outcome variables $(n=2706)$

\begin{tabular}{|c|c|c|c|}
\hline Variable & Full sample & $\begin{array}{r}\text { Parent } \\
n=1353\end{array}$ & $\begin{array}{l}\text { Adolescent } \\
n=1353\end{array}$ \\
\hline Age & & $43.7 S D=7.5$ & $14.5 S D=1.6$ \\
\hline Percent Female & $1688(62 \%)$ & $1006(74 \%)$ & $682(50 \%)$ \\
\hline Body Mass Index & & $28.07 S D=7.2$ & $20.63 S D=8.3$ \\
\hline Underweight & & $14(1 \%)$ & $70(4 \%)$ \\
\hline Normal weight & & $474(35 \%)$ & $1120(65 \%)$ \\
\hline Overweight & & $405(30 \%)$ & $248(14 \%)$ \\
\hline Obese & & $406(30 \%)$ & $203(12 \%)$ \\
\hline \multicolumn{4}{|l|}{ Education } \\
\hline Less than high school & & $18(1 \%)$ & \\
\hline High school & & $230(17 \%)$ & \\
\hline Some college & & $468(35 \%)$ & \\
\hline College & & $637(47 \%)$ & \\
\hline $6^{\text {th }}$ grade or less & & & $95(7 \%)$ \\
\hline $7^{\text {th }}$ grade & & & $216(16 \%)$ \\
\hline $8^{\text {th }}$ grade & & & $230(17 \%)$ \\
\hline $9^{\text {th }}$ grade & & & $216(16 \%)$ \\
\hline $10^{\text {th }}$ grade & & & $271(20 \%)$ \\
\hline $11^{\text {th }}$ grade & & & $257(19 \%)$ \\
\hline $12^{\text {th }}$ grade & & & $68(5 \%)$ \\
\hline \multicolumn{4}{|l|}{ Race } \\
\hline American Indian/ Alaskan & $63(2 \%)$ & $24(2 \%)$ & $39(3 \%)$ \\
\hline Asian & $118(4 \%)$ & $53(4 \%)$ & $65(5 \%)$ \\
\hline Black & $487(18 \%)$ & $228(17 \%)$ & $259(19 \%)$ \\
\hline Pacific Islander/ Hawaiian & $9(<1 \%)$ & $3(<1 \%)$ & $6(1 \%)$ \\
\hline White & $2135(79 \%)$ & $1069(77 \%)$ & $1066(80 \%)$ \\
\hline Hispanic/Latino(a) & $236(9 \%)$ & $100(8 \%)$ & $136(10 \%)$ \\
\hline
\end{tabular}

Note: Adolescent weight categories reported are based on adolescent weight percentile, rather than by the standard cutpoints used for adult weight categories based on BMI 
Table 2. Means and Standard Deviations for parent instrumentality and health behaviors

\begin{tabular}{lrr}
\hline & \multicolumn{1}{c}{ Parent } & \multicolumn{1}{c}{ Adolescent } \\
\hline Fruit and vegetable instrumentality & $3.91(0.69)$ & $3.76(0.82)$ \\
Hedonic food instrumentality & $3.53(0.69)$ & $3.30(0.78)$ \\
Exercise instrumentality & $3.34(0.85)$ & $3.14(0.93)$ \\
Sedentariness instrumentality & $3.33(0.68)$ & $3.02(0.70)$ \\
Fruit and vegetable consumption & $2.21(1.62)$ & $1.84(1.55)$ \\
Hedonic food consumption & $3.73(3.66)$ & $5.05(4.15)$ \\
Exercise & $178.29(178.11)$ & $278.54(13.10)$ \\
Sedentariness & $380.02(221.63)$ & $111.83(18.01)$ \\
\hline
\end{tabular}


Table 3. Preliminary analyses examining associations of potential mediators with BMI

\begin{tabular}{lrrrrrr}
\hline & \multicolumn{3}{c}{ Adolescent BMI } & \multicolumn{3}{c}{ Parent BMI } \\
\cline { 2 - 7 } & \multicolumn{1}{c}{$\beta$} & $95 \%$ CI & $p$ & $\beta$ & $95 \%$ CI & $p$ \\
\cline { 2 - 7 } Adolescent F\&V & -0.04 & $-0.09,0.01$ & .145 & -- & -- & -- \\
Adolescent Hedonic & -0.01 & $-0.08,0.07$ & .951 & -- & -- & -- \\
Adolescent Exercise & -0.23 & $-0.27,-0.19$ & $<.001$ & -- & -- & -- \\
Adolescent Sedentary & 0.17 & $0.13,0.22$ & $<.001$ & -- & -- & -- \\
Parent F\&V & -- & -- & -- & -0.09 & $-0.14,-0.04$ & .002 \\
Parent Hedonic & -- & -- & -- & 0.20 & $0.09,0.32$ & .004 \\
Parent Exercise & -- & -- & -- & 0.02 & $-0.04,0.08$ & .667 \\
Parent Sedentary & -- & -- & -- & 0.16 & $0.12,0.20$ & $<.001$ \\
\hline
\end{tabular}


Table 4. Final APIM with direct and indirect associations of instrumentality with behaviors and BMI

\begin{tabular}{|c|c|c|c|c|c|c|c|c|c|c|c|c|c|c|c|c|c|c|}
\hline \multirow[b]{3}{*}{$\mathbf{F} \& \mathbf{V}$} & \multicolumn{3}{|c|}{ Adolescent Behavior } & \multicolumn{3}{|c|}{ Parent Behavior } & \multicolumn{6}{|c|}{ Adolescent BMI } & \multicolumn{6}{|c|}{ Parent BMI } \\
\hline & \multicolumn{6}{|c|}{ Direct Paths } & \multicolumn{3}{|c|}{ Direct Paths } & \multicolumn{3}{|c|}{$\begin{array}{l}\text { Indirect paths via adolescent } \\
\text { behavior }\end{array}$} & \multicolumn{3}{|c|}{ Direct Paths } & \multicolumn{3}{|c|}{$\begin{array}{l}\text { Indirect paths via parent } \\
\text { behavior }\end{array}$} \\
\hline & $\beta$ & $95 \% C I$ & $p$ & $\beta$ & $95 \% C I$ & $p$ & $\beta$ & $95 \% C I$ & $p$ & $\beta$ & $95 \% C I$ & $p$ & $\beta$ & $95 \% C I$ & $p$ & $\beta$ & $95 \% C I$ & $p$ \\
\hline APPI & 0.13 & $0.12,0.23$ & $<.001$ & 0.13 & $0.07,0.18$ & $<.001$ & -0.07 & $-0.14,-0.01$ & .071 & - & - & - & 0.02 & $-0.05,0.08$ & .658 & -0.01 & $-0.01,0.00$ & .103 \\
\hline PPSI & 0.17 & $0.08,0.19$ & $<.001$ & 0.22 & $0.17,0.28$ & $<.001$ & -0.04 & $-0.10,0.02$ & .315 & - & - & - & -0.03 & $-0.10,0.03$ & .395 & -0.01 & $-0.02,-0.00$ & .084 \\
\hline \multicolumn{19}{|c|}{ Hedonic } \\
\hline APPI & -0.08 & $-0.09,0.02$ & .331 & 0.02 & $-0.04,0.08$ & .539 & $<0.01$ & $-0.06,0.07$ & .959 & - & 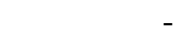 & - & 0.06 & $-0.01,0.12$ & .076 & $<0.01$ & $-0.00,0.01$ & .546 \\
\hline PPSI & -0.03 & $-0.13,-0.02$ & .028 & -0.12 & $-0.18,-0.07$ & $<.001$ & 0.08 & $0.02,0.14$ & .027 & - & - & - & -0.02 & $-0.08,0.05$ & .127 & -0.01 & $-0.02,-0.01$ & .014 \\
\hline \multicolumn{19}{|c|}{ Exercise } \\
\hline APPI & 0.09 & $0.06,0.12$ & $<.001$ & 0.10 & $0.01,0.18$ & .042 & 0.08 & $0.02,0.15$ & .035 & -0.02 & $-0.03,-0.01$ & $<.001$ & -0.09 & $-0.15,-0.03$ & .023 & - & - & 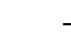 \\
\hline PPSI & 0.05 & $0.01,0.08$ & .017 & 0.05 & $-0.03,0.13$ & .281 & 0.09 & $0.03,0.15$ & .016 & -0.01 & $-0.01,-0.00$ & .023 & -0.07 & $-0.13,-0.01$ & .076 & - & - & - \\
\hline \multicolumn{19}{|c|}{ Sedentary } \\
\hline APPI & -0.07 & $-0.12,-0.02$ & .025 & -0.06 & $-0.12,-0.00$ & .093 & -0.16 & $-0.22,-0.09$ & $<.001$ & 0.00 & $-0.01,0.00$ & .163 & $<0.01$ & $-0.06,0.06$ & .979 & -0.01 & $-0.01,0.00$ & .045 \\
\hline PPSI & -0.02 & $-0.08,0.03$ & .478 & -0.08 & $-0.14,-0.02$ & .027 & -0.01 & $-0.07,0.05$ & .728 & 0.00 & $-0.00,0.00$ & .509 & 0.01 & $-0.05,0.07$ & .723 & -0.01 & $-0.01,-0.00$ & .115 \\
\hline
\end{tabular}

APPI = Adolescent-perceived parent instrumentality

PPSI $=$ Parent-perceived self-instrumentality 


\section{References}

1. Fryar CD, Carroll MD, Ogden, CL. Prevalence of overweight and obesity among children and adolescents: United States, 1963-1965 through 2011-2012. Center for Disease Control and Prevention, Division of Health and Nutrition Examination Surveys. 2016.

2. Ogden CL, Carroll MD, Lawman HG, et al. Trends in obesity prevalence among children and adolescents in the United States, 1988-1994 through 2013-2014. JAMA. 2016; 315(21): 2292-2299.

3. Whitlock EP, O'Connor EA, Williams SB, Beil TL, Lutz KW. Effectiveness of Primary Care Interventions for Weight Management Children Adolescents: An Updated, Targeted Systematic Review for the USPSTF. Agency for Healthcare Research and Quality (US). 2010. 4. Healthypeople.gov. Healthy People 2020. Available at https://www.healthypeople.gov/2020/topics-objectives. Accessibility verified August, 30, 2017. 5. Orehek E, Forest AL, Barbaro N. A people-as-means approach to interpersonal relationships. Perspect Psychol Sci. 2018 May;13(3):373-89.

6. Orehek E, Forest AL. When people serve as means to goals: Implications of a motivational account of close personal relationships. Curr Dir Psychol Sci. 2006; 25: 79-84. 7. Delormier T, Frohlich KL, Potvin L. Food and eating as social practice-understanding eating patterns as social phenomena and implications for public health. Sociol Health Illn. 2009; 31(2): 215-228.

8. Hsu CL, Lu HP. Why do people play on-line games? An extended TAM with social influences and flow experience. Inf \& Manag. 2004; 41(7): 853-868.

9. Hoefer WR, McKenzie TL, Sallis JF, Marshall SJ, Conway TL. Parental provision of transportation for adolescent physical activity. Am J Prev Med. 2001; 21(1): 48-51.

10. Hofmann W, Finkel EJ, Fitzsimons GM. Close relationships and self-regulation: How relationship satisfaction facilitates momentary goal pursuit. J Pers Soc Psychol. 2015; 109(3): 434-452.

11. Overall NC, Fletcher GJ, Simpson JA. Helping each other grow: Romantic partner support, self-improvement, and relationship quality. Pers Soc Psychol Bull. 2010; 36(10): 14961513.

12. Anderson ES, Winett RA, Wojcik JR. Self-regulation, self-efficacy, outcome expectations, and social support: social cognitive theory and nutrition behavior. Ann Behav Med. 2007; 34(3): 304-312.

13. Duncan SC, Duncan TE, Strycker LA. Sources and types of social support in youth physical activity. Health Psychol. 2005; 24(1): 3-10.

14. Steptoe A, Perkins-Porras L, Rink E, Hilton S, Cappuccio FP. Psychological and social predictors of changes in fruit and vegetable consumption over 12 months following behavioral and nutrition education counseling. Health Psychol. 2004; 23(6): 574.

15. Neumark-Sztainer D, Wall M, Perry C, Story M. Correlates of fruit and vegetable intake among adolescents: Findings from Project EAT. Prev Med. 2003; 37(3): 198-208.

16. Lopez NV, Ayala GX, Corder K, et al. Parent support and parent-mediated behaviors are associated with children's sugary beverage consumption. J Acad Nutr Diet. 2012; 112(4): 541547.

17. Morrison H, Power TG, Nicklas T, Hughes SO. Exploring the effects of maternal eating patterns on maternal feeding and child eating. Appetite. 2013; 63: 77-83.

18. Oh AY, Davis T, Dwyer LA, Hennessy E, L, T, Yaroch AL, Nebeling LC. Recruitment, 
enrollment, and response of parent-adolescent dyads in the FLASHE study. Am J Prev Med; 52(6): 849-855.

19. Bolger N, Zuckerman A, Kessler RC. Invisible support and adjustment to stress. J Pers Soc Psychol. 2000; 79(6): 953-961.

20. Girme YU, Overall NC, Simpson JA. When visibility matters: Short-term versus longterm costs and benefits of visible and invisible support. Pers Soc Psychol Bull. 2013; 39(11): 1441-1454.

21. Nebeling LC, Hennessey EM, Oh AY, et al. The FLASHE Study: Survey development, dyadic perspectives, and participant characteristics. Am J Prev Med. 2017; 52(6): 839-848.

22. Alaimo K, Olson CM, Frongillo EA. Importance of cognitive testing for survey items: an example from food security questionnaires. J Nutr Educ Behav. 1999 Sep 1;31(5):269-75.

23. Collins D. Pretesting survey instruments: an overview of cognitive methods. Qual Life Res. 2003 May 1;12(3):229-38.

24. Birch LL, Fisher JO, Grimm-Thomas K, Markey CN, Sawyer R, Johnson SL.

Confirmatory factor analysis of the Child Feeding Questionnaire: a measure of parental attitudes, beliefs and practices about child feeding and obesity proneness. Appetite. 2001; 36(3): 201-210.

25. Darling N, Cumsille P, Martínez ML. Individual differences in adolescents' beliefs about the legitimacy of parental authority and their own obligation to obey: A longitudinal investigation. Child Dev. 2008; 79(4): 1103-1118.

26. Davison KK, Li K, Baskin ML, Cox T, Affuso O. Measuring parental support for children's physical activity in white and African American parents: The Activity Support Scale for Multiple Groups. Prev Med. 2011; 52(1): 39-43.

27. Larios SE, Ayala GX, Arredondo EM, Baquero B, Elder JP. Development and validation of a scale to measure Latino parenting strategies related to children's obesigenic behaviors. The parenting strategies for eating and activity scale. Appetite. 2009; 52(1): 166-172.

28. Musher-Eizenman D, Holub S. Comprehensive Feeding Practices Questionnaire: validation of a new measure of parental feeding practices. J Pediatr Psychol. 2007; 32(8): 960972.

29. Wardle J, Sanderson S, Guthrie CA, Rapoport L, Plomin R. Parental feeding style and the inter-generational transmission of obesity risk. Obes Res. 2002; 10(6): 453-462.

30. National Cancer Institute. Dietary Assessment Primer: Screeners at a Glance. Available at http://dietassessmentprimer.cancer.gov/profiles/screeners/. Accessibility verified October 28, 2015.

31. Brener ND, Merlo C, Eaton D, Kann L, Park S, Blanck HM. Beverage Consumption Among High School Students-United States, 2010. JAMA. 2011; 306(4): 369-371. (Reprinted from MMWR. 2011; 60: 778-780).

32. Ford ES, Zhao G, Tsai J, Li C. Low-risk lifestyle behaviors and all-cause mortality: findings from the National Health and Nutrition Examination Survey III Mortality Study. Am J Public Health. 2011; 101(10): 1922-1929.

33. Smith TM, Calloway EE, Pinard CA, et al. Using secondary 24-hour dietary recall data to estimate daily dietary factor intake from the FLASHE study dietary screener. Am J Prev Med. 2017; 52(6): 856-862.

34. Block G, Gillespie C, Rosenbaum EH, Jenson C. A rapid food screener to assess fat and fruit and vegetable intake. Am J Prev Med. 2000; 18(4): 284-288.

35. Andersen LF, Johansson L, Solvoll K. Usefulness of a short food frequency questionnaire 
for screening of low intake of fruit and vegetable and for intake of fat. Eur J Public Health. 2002; 12(3): 208-213.

36. Nelson MC, Lytle LA. Development and evaluation of a brief screener to estimate fastfood and beverage consumption among adolescents. J Amer Diet Assoc. 2009; 109(4): 730-734. 37. Saint-Maurice PF, Kim Y, Hibbing P, Oh AY, Perna FM, Welk GJ. Calibration and validation of the Youth Activity Profile: the FLASHE study. Am J Prev Med. 2017; 52(6): 880887.

38. Craig CL, Marshall AL, Sjostrom M, Bauman AE, Booth ML, Ainsworth BE, Pratt M, Ekelund U, Yngve A, Sallis JF, Oja P. International physical activity questionnaire: 12- country reliability and validity. Med Sci Sports Exerc. 2003;35(8):1381-1395.

39. Cerin E, Cain KL, Oyeyemi AL, Owen N, Conway TL, Cochrane T, Van Dyck D, Schipperijn J, Mitáš J, Toftager M, Aguinaga-Ontoso I. Correlates of agreement between accelerometry and self-reported physical activity. Med Sci Sports Exerc. 2016 Jun;48(6):10751084.

40. Lee PH, Macfarlane DJ, Lam TH, Stewart SM. Validity of the international physical activity questionnaire short form (IPAQ-SF): A systematic review. Int J Behav Nutr Phys Act. 2011 Dec;8(1):115.

41. National Cancer Institute (NCI). 2014 Family Life, Activity, Sun, Health and Eating (FLASHE) Survey Data. Rockville, MD: U.S. Department of Health and Human Services, National Cancer Institute. http://cancercontrol.cancer.gov/brp/hbrb/flashe.html

42. Westat, Inc. Family Life, Activity, Sun, Health, and Eating (FLASHE) Study Methodology Report. Published July 2015.

43. Ferrer RA, Green PA, Oh AY, Hennessy E, Dwyer LA. Emotion Suppression, Emotional Eating, and Eating Behavior Among Parent-Adolescent Dyads. Emotion. 2017.

44. Campbell L, Kashy DA. Estimating actor, partner, and interaction effects for dyadic data using PROC MIXED and HLM: A user-friendly guide. Pers Relationships. 2002; 9(3): 327-342. 45. Cook WL, Kenny DA. The actor-partner interdependence model: A model of bidirectional effects in developmental studies. Int J of Behav Devel. 2005; 29(2):101-109.

46. MacKinnon DP, Fairchild AJ, Fritz MS. Mediation analysis. Annu. Rev. Psychol. 2007 Jan 10;58:593-614.

47. Enders, CK. Applied missing data analysis. 2010. New York: Guilford Press

48. Kline, RB. Principles and practices of structural equation modeling, Third Edition. 2004. New York: Guilford.

49. Prochaska JJ, Spring B, Nigg CR. Multiple health behavior change research: an introduction and overview. Prev Med. 2008 Mar 1;46(3):181-8.

50. Fitzsimons GM, Finkel EJ, Vandellen MR. Transactive goal dynamics. Psychol Rev. 2015; 122(4): 648-673.

51. Inagaki TK, Orehek E. On the benefits of giving social support: When, why, and how support providers gain by caring for others. Curr Dir Psychol Sci. 2017; 26(2): 109-113.

52. Converse BA, Fishbach A. Instrumentality boosts appreciation: Helpers are more appreciated while they are useful. Psychol Sci. 2012; 23: 560-566.

53. Fitzsimons GM, Fishbach, A. Shifting closeness: interpersonal effects of personal goal progress. J Pers Soc Psychol. 2010; 98: 535-549.

54. Fitzsimons GM, Shah J. How goal instrumentality shapes relationship evaluations. J Pers Soc Psychol. 2008; 95: 319-337. 
55. Orehek E, Forest AL, Wingrove, S. People as means to multiple goals: Implications for interpersonal relationships. Pers Soc Psychol Bull. In Press.

56. Stokols D, Allen J, Bellingham RL. The social ecology of health promotion: implications for research and practice. Am J Health Promot. 1996 Mar;10(4):247-51. 